\title{
Central Contact for Recruitment Backup
}

National Cancer Institute

\section{Source}

National Cancer Institute. Central Contact for Recruitment Backup. NCI Thesaurus. Code C127524.

The secondary contact person responsible for coordinating recruitment information for a study. 